\title{
SPRINGER'S ODD DEGREE EXTENSION THEOREM FOR QUADRATIC FORMS OVER SEMILOCAL RINGS
}

\author{
PHILIPPE GILLE AND ERHARD NEHER
}

In memory of T.A. Springer

\begin{abstract}
A fundamental result of Springer says that a quadratic form over a field of characteristic $\neq 2$ is isotropic if it is so after an odd degree field extension. In this paper we generalize Springer's theorem as follows. Let $R$ be an arbitrary semilocal ring, let $S$ be a finite $R$-algebra of constant odd degree, which is étale or generated by one element, and let $q$ be a nonsingular $R$-quadratic form whose base ring extension $q_{S}$ is isotropic. We show that then already $q$ is isotropic.
\end{abstract}

Keywords: Quadratic forms, semilocal rings, Springer Odd Degree Extension Theorem.

MSC 2000: 11E08, 11E81.

\section{INTRODUCTION}

A celebrated result of Springer [Sp] says that a quadratic form over a field $F$ of characteristic $\neq 2$ that becomes isotropic over an odd degree field extension is already isotropic over $F$. The result was conjectured by Witt and was also proven, but not published by E. Artin. An account of Springer's result is given in [Lam, VII, Thm. 2.7] or [Sch, II, Thm. 5.3]. It is proven for arbitrary fields in [EKM, 18.5].

Springer's Theorem has many important consequences, for example for Witt groups. It is therefore not surprising that it has been generalized by replacing odd degree extensions by more general field extensions, see for example, [Ho1, Prop. 5.3], [Ho2, Cor. 4.2] or [Lag, Lem. 2.8], or by replacing the base field $F$ by more general rings. The main result of this paper goes in the latter direction:

Date: June 18, 2021.

P. Gille was supported by the ANR project Geolie ANR-15-CE40-0012.

E. Neher acknowledges partial support from NSERC through a Discovery grant.

This work was supported by the Labex Milyon (ANR-10-LABX-0070) of Université de Lyon, within the program "Investissements d'Avenir" (ANR-11-IDEX-0007) operated by the French National Research Agency (ANR). 
Odd Degree Extension Theorem. (Theorem 2.1) Let $R$ be a semilocal ring, $M$ a finite projective $R$-module, $q: M \rightarrow R$ a nonsingular quadratic form, and $S$ a finite $R$-algebra of constant odd degree, which is étale or one-generated. If the base ring extension $q_{S}$ is isotropic, then already $q$ is isotropic.

Since the terminology regarding quadratic forms over rings is not standard, see the comparison in 1.2(a), let us explain what we mean with a nonsingular quadratic form. Recall that the radical of $(M, q)$ with polar form $b_{q}$ is $\operatorname{rad}(M, q)=\left\{m \in M: q(m)=0=b_{q}(m, M)\right\}$. We call $q$ nonsingular, if $\operatorname{rad}\left((M, q)_{F}\right)=\{0\}$ for all $R$-fields $F$. An example of such a form is a regular quadratic form, defined by the condition that its polar form induces an isomorphism between $M$ and its dual space. Nonsingular and regular quadratic forms are the same in case $2 \in R^{\times}=\{u \in R: u R=R\}$, the units of $R$, or in case the projective $R$-module $M$ has constant even rank, but not in general. For example, for $u \in R^{\times}$the quadratic form $R \rightarrow R$, $x \mapsto u x^{2}$ is always nonsingular, but regular only if $2 \in R^{\times}$. We call a finite projective $R$-algebra $S$ of constant degree $d$ one-generated if it is generated by one element, equivalently, $S \cong R[X] / f$ for a monic polynomial of degree $d$.

Special cases of the Odd Degree Extension Theorem have been proven before; all of them assumed $2 \in R^{\times}$, so that nonsingular $=$regular. Specifically, it was proven by Panin and Rehmann [PR] under the assumption that $R$ is a noetherian local integral domain, which has an infinite residue field, and that $S$ is étale (necessarily one-generated in this case). It was extended in $[\mathrm{PP}]$ by Panin-Pimenov to $R$ a semi-local noetherian integral domain whose residue fields are all infinite. It was stated in [Scu] for $R$ semilocal, $2 \in R^{\times}$and $S$ finite étale of constant odd degree. However, there is a serious gap in the proof of the crucial Proposition 3.1 of [Scu]. As a result, the Odd Degree Extension Theorem, the main theorem of [Scu], is not proven in the generality stated.

For proving the Odd Degree Extension Theorem one can easily reduce to $M$ of constant rank $r \geq 2$. The case $r=2$ (Lemma 2.2) holds for any finite $R$-algebra; our proof uses a consequence of Deligne's trace homomorphism for the flat cohomology of abelian affine group schemes (Lemma B.3). For $r \geq 3$ we follow an approach inspired by the paper [PR] of Panin and Rehmann and first deal with one-generated algebras. A crucial step here is proving that isotropic elements can be lifted from $M \otimes_{R}(S / \operatorname{Jac}(R) S)$ to $M \otimes_{R} S$ (Corollary A.6). We obtain this as an application of Demazure's Conjugacy Theorem for reductive $R$-group schemes. We should point out, that in this part we use the classical version of the Odd Degree Extension Theorem ( $R$ a field, $[\mathrm{EKM}])$. Once the case of one-generated algebras has been settled, the case of étale algebras easily follows by applying a recent result of Bayer-Fluckiger, First and Parimala $[\mathrm{BFP}]$ on embeddings of finite étale $R$-algebras into one-generated $R$-algebras. 
Structure of the paper. Section 1 presents a review of nonsingular quadratic forms and proves some results needed for our proof of the Odd Degree Extension Theorem in the first part of section 2. In the second part of that section we prove some consequences of the theorem well-known in case of base fields. The paper closes with two appendices, A and B. Their results are used in our proof of the Odd Degree Extension Theorem, but they are of interest beyond that Theorem.

Basic notation. Throughout, $R$ is a commutative associative unital ring. We do not assume that $2 \in R^{\times}$, unless this is explicitly so stated. We denote by $\operatorname{Specmax}(R)$ the subspace of the spectrum $\operatorname{Spec}(R)$ whose elements are the maximal ideals of $R$. Modules, quadratic forms and algebras will be defined over $R$. We use $R$-alg to denote the category of commutative associative unital $R$-algebras. Objects of $R$-alg are sometimes also referred to as $R$-rings. If $M$ is an $R$-module and $S \in R$-alg, we put $M_{S}=M \otimes_{R} S$.

We call a submodule $U$ of an $R$-module $M$ complemented if there exists a submodule $U^{\prime}$ of $M$ such that $U \oplus U^{\prime}=M$. We say an $R$-module $M$ is finite projective if it is finitely generated projective (= locally free of finite rank). A finite $R$-algebra is an algebra $S \in R$-alg whose underlying $R$-module is finitely generated, but not necessarily projective. A finite projective $R-$ algebra is an $R$-ring $S$ whose underlying $R$-module is finite projective. The norm of such an $R$-algebra $S$ is denoted $\mathrm{N}_{S / R}$. A finite projective $R$-algebra $S$ is called finite étale if it is separable, see for example [Fo] or [Knu] for other equivalent definitions. We call $S \in R$-alg an étale $R$-algebra of degree $d \in \mathbb{N}$ if $S$ is a finite étale algebra whose underlying $R$-module is projective of constant rank $d$.

\section{QuADRATIC FORMS}

This section starts with a review of known facts on bilinear and quadratic forms over arbitrary rings, thereby also establishing our notation and terminology, 1.1-1.3. We study quadratic forms over semilocal rings in the second part of this section.

1.1. Quadratic and symmetric bilinear forms (terminology, basic facts). (a) A (symmetric) bilinear module is a pair $(M, b)$ consisting of a finite projective $R$-module and a symmetric $R$-bilinear map $b: M \times M \rightarrow R$. Except when otherwise stipulated, we will only consider symmetric bilinear forms. It is therefore convenient to simply speak of bilinear forms and bilinear modules. We use the symbol $(M, b) \cong\left(M^{\prime}, b^{\prime}\right)$ to denote isometric bilinear modules. When $M$ is clear from the context or unimportant, we will sometimes write $b$ for $(M, b)$. Given a bilinear module $(M, b)$, its adjoint is the $R$-linear map $b^{*}: M \rightarrow M^{*}=\operatorname{Hom}_{R}(M, R), m \mapsto b(m, \cdot)$. We call $(M, b)$ regular if $b^{*}$ is an isomorphism.

(b) A quadratic module is a pair $(M, q)$ where $M$ is a finite projective $R$-module and $q: M \rightarrow R$ is an $R$-quadratic form. We use $b_{q}$ to denote the polar form of $q$. We call $q$ or $(M, q)$ regular if $b_{q}$ is regular. As for 
bilinear modules, $(M, q) \cong\left(M^{\prime}, q^{\prime}\right)$ indicates isometric quadratic modules. We sometimes write $q$ instead of $(M, q)$ if $M$ is unimportant or clear from the context.

(c) (Base change) Let $S \in R$-alg, and let $(M, b)$ be an $R$-bilinear module. There exists a unique $S$-bilinear form $b_{S}: M_{S} \times M_{S} \rightarrow S$ satisfying $b_{S}\left(m_{1} \otimes s_{1}, m_{2} \otimes s_{2}\right)=b\left(m_{1}, m_{2}\right) s_{1} s_{2}$ for $m_{i} \in M$ and $s_{i} \in S$. Given an $R$-quadratic module $(N, q)$, there exists a unique $S$-quadratic form $q_{S}: N_{S} \rightarrow S$ satisfying $q_{S}(n \otimes s)=q(n) s^{2}$ for $s \in S$ and $n \in N$. The polar of $q_{S}$ is the base change of the polar of $q$. If $(M, b)($ or $(N, q))$ is regular, then so is $\left(M_{S}, b_{S}\right)$ (respectively $\left(N_{S}, q_{S}\right)$ ).

(d) (Tensor products) Let $\left(M_{i}, b_{i}\right), i=1,2$, be bilinear modules. There exists a unique symmetric bilinear form $b_{1} \otimes b_{2}$ on $M_{1} \otimes_{R} M_{2}$ satisfying $\left(b_{1} \otimes b_{2}\right)\left(m_{1} \otimes m_{2}, m_{1}^{\prime} \otimes m_{2}^{\prime}\right)=b_{1}\left(m_{1}, m_{1}^{\prime}\right) b_{2}\left(m_{2}, m_{2}^{\prime}\right)$ for $m_{i}, m_{i}^{\prime} \in M_{i}$. Given an $R$-bilinear module $(M, b)$ and an $R$-quadratic form $(N, q)$, there exists a unique $R$-quadratic form $b \otimes q: M \otimes_{R} N \rightarrow R$ satisfying

$$
\begin{aligned}
(b \otimes q)(m \otimes n) & =b(m, m) q(n), \text { and } \\
b_{b \otimes q}\left(m \otimes n, m^{\prime} \otimes n^{\prime}\right) & =b\left(m, m^{\prime}\right) b_{q}\left(n, n^{\prime}\right)
\end{aligned}
$$

for all $m, m^{\prime} \in M$ and $n, n^{\prime} \in N$, where $b_{q}$ is the polar form of $q$. It is called the tensor product of $(M, b)$ and $(N, q)$, [Sa, Thm. 1]. The polar form of $b \otimes q$ is the tensor product of the symmetric bilinear forms $b \otimes b_{q}$. If $(M, b)$ and $(N, q)$ are regular, then so is $(M, b) \otimes(N, q)$. The tensor product is compatible with base change: for $S \in R$-alg we have

$$
(M, b)_{S} \otimes_{S}(N, q)_{S} \stackrel{\sim}{\longrightarrow}\left((M, b) \otimes_{R}(N, q)\right)_{S}
$$

with respect to $m \otimes s_{1} \otimes n \otimes s_{2} \mapsto m \otimes m \otimes s_{1} s_{2}$.

(e) Given a bilinear module $(M, b)$, the orthogonal module of a submodule $U \subset M$ is $U^{\perp}=\{m \in M: b(m, U)=0\}$. We call $U$ totally isotropic if $U \subset U^{\perp}$. A submodule $U$ of a quadratic module $(M, q)$ is totally isotropic if $q(U)=0$, in which case $U$ is also a totally isotropic submodule of $\left(M, b_{q}\right)$. If $(M, b)$ is a bilinear module and $U \subset M$ is a submodule such that $\left(U,\left.b\right|_{U}\right)$ is regular, then $M=U \oplus U^{\perp}$.

1.2. Nonsingular quadratic forms. Recall that the radical of a quadratic module $(M, q)$ is $\operatorname{rad}(M, q)=\left\{m \in M: q(m)=0=b_{q}(m, M)\right\}$. It is a submodule of $M$ and satisfies $(\operatorname{rad}(M, q))_{S} \subset \operatorname{rad}\left((M, q)_{S}\right), S \in R$-alg. We call $(M, q)$ or simply $q$ nonsingular if $\operatorname{rad}\left((M, q)_{F}\right)=\{0\}$ for all fields $F \in$ $R$-alg. A quadratic space is a quadratic module $(M, q)$ with a nonsingular $q$. We will use the following properties of nonsingular forms (for details see the references in (a)).

(a) (Comparison of terminology) Our terminology of a regular bilinear form or regular quadratic form follows [Knu] and [Sch] (except that in these references "regular" and "nonsingular" are used interchangeably), but a regular bilinear form as defined here is called "non singular" in [Ba] and 
"nondegenerate" in [EKM]. A nonsingular quadratic form is called "nondegenerate" in [Co1], "nondegenerate" in [EKM], "semiregular" in [Knu] in case of constant odd rank, cf. (e), and "separable" in [Pe].

(b) A regular quadratic form is nonsingular, since $\operatorname{rad}\left((M, q)_{S}\right)=0$ for a regular form and any $S \in R$-alg by $1.1(\mathrm{c})$.

(c) If $2 \in R^{\times}$, a nonsingular form is regular. Indeed, if $2 \in R^{\times}$, then $\operatorname{rad}\left(q_{S}\right)=\left\{m \in M_{S}: b_{q_{S}}\left(m, M_{S}\right)=0\right\}, S \in R$-alg, and so the adjoint of $b_{q}$ is an isomorphism by Nakayama.

(d) If $q$ is nonsingular, then so is $q_{S}$ for any $S \in R$-alg.

(e) Let $(M, q)$ be a quadratic module with $M$ of constant even rank. Then $q$ is nonsingular if and only if $q$ is regular, cf. (h) below.

(f) A quadratic $R$-space $(M, q)$ with $M$ faithfully projective is primitive in the sense that $q(M)$ generates $R$ as ideal. If $R$ is semilocal, then $q(m) \in R^{\times}$ for some $m \in M$ (which is unimodular in the sense of 1.8).

(g) Let $(M, q)=\left(M_{1}, q_{1}\right) \perp\left(M_{2}, q_{2}\right)$ be a direct sum of quadratic modules. If $q$ is nonsingular, then so are $q_{1}$ and $q_{2}$. Conversely, if $q_{1}$ is regular, then $q$ is nonsingular if and only if $q_{2}$ is nonsingular. If $q_{1}$ and $q_{2}$ are nonsingular, then $q$ need not be nonsingular, see the example in 1.7 .

(h) (1-dimensional forms) Let $u \in R^{\times}$. We define $\langle u\rangle_{b}: R \times R \rightarrow R$, $\left(r_{1}, r_{2}\right) \mapsto u r_{1} r_{2}$ and $\langle u\rangle_{q}: R \rightarrow R, r \mapsto u r^{2}$. Then $\langle u\rangle_{b}$ is a regular bilinear form. The polar of the quadratic form $\langle u\rangle_{q}$ is $2\langle u\rangle$, whence $\langle u\rangle_{q}$ is regular if and only if $2 \in R^{\times}$, but $\langle u\rangle_{q}$ is always nonsingular. We abbreviate $\langle u\rangle=\langle u\rangle_{b}$ if the meaning of $\langle u\rangle$ is clear from the context.

If $(M, b)$ and $(N, q)$ are bilinear and quadratic modules respectively, then

$$
\langle 1\rangle_{b} \otimes b \cong b, \quad\langle 1\rangle_{b} \otimes q \cong q
$$

under the standard isomorphism $R \otimes_{R} M \stackrel{\sim}{\longrightarrow} M$. However, $b \otimes\langle 1\rangle_{q}=q_{b}$, where $q_{b}: M \rightarrow R, m \mapsto b(m, m)$, is the quadratic form associated with $b$. Its polar is $b_{q_{b}}=2 b$.

(i) (Reduction to constant rank) Let $R=R_{0} \times \cdots \times R_{n}$ be a direct product of rings. For $0 \leq i \leq n$ we view $e_{i}=1_{R_{i}}$ as an idempotent of $R$. Thus $\left(e_{0}, \ldots, e_{n}\right)$ is a system of mutually orthogonal idempotents with $R_{i}=R e_{i}$. Any quadratic $R$-module $(M, q)$ uniquely decomposes into the orthogonal sum of quadratic $R$-modules

$$
(M, q)=\left(M_{0}, q_{0}\right) \perp \cdots \perp\left(M_{n}, q_{n}\right),
$$

where $M_{i}=M e_{i}$ and $q_{i}$ is restriction of $q$ to $M_{i}$. By a slight abuse of notation we will also consider $\left(M_{i}, q_{i}\right)$ as a quadratic $R_{i}$-module and identify $(M, q)$ with the direct product $\left(M_{1}, q_{1}\right) \times \cdots \times\left(M_{n}, q_{n}\right)$ of quadratic $R_{i}-$ modules. Conversely, given quadratic $R_{i}$-modules $\left(M_{i}, q_{i}\right), 0 \leq i \leq n$, we obtain a quadratic $R$-module $(M, q)$ for which (1.2.1) holds. The quadratic $R$-module $(M, q)$ is regular (resp. nonsingular) if and only if all quadratic $R_{i}$-modules $\left(M_{i}, q_{i}\right)$ are regular (resp. nonsingular). 
For arbitrary $R$, a finite projective $R$-module $M$ gives rise to a decomposition $R=R_{0} \times \cdots \times R_{n}$ and hence to $M=M_{0} \times \cdots \times M_{n}$ such that $M_{i}$ is a projective $R_{i}$-module of constant rank $i$. The discussion above then describes the reduction of quadratic modules to quadratic modules of constant rank.

(j) (Witt cancellation) Let $R$ be a semilocal ring, let $q_{1}, q_{2}, q_{1}^{\prime}$ and $q_{2}^{\prime}$ be quadratic forms where $q_{1}$ is regular and $q_{2}$ is nonsingular and of positive rank. If $q_{1} \cong q_{1}^{\prime}$ and $q_{1} \perp q_{2} \cong q_{1}^{\prime} \perp q_{2}^{\prime}$, then $q_{2} \cong q_{2}^{\prime}$. Indeed, since the nonsingular form $q_{2}$ is primitive by (f), this follows from [Kne, Kürzungssatz]. Witt cancellation with all forms $q_{i}$ and $q_{i}^{\prime}$ being regular, is proven in [Ba, III, (4.3)].

1.3. Metabolic and hyperbolic spaces. Let $(U, b)$ be a bilinear module. The metabolic space associated with $(U, b)$ is the bilinear module $\mathbb{M}(U, b)=$ $\left(U \oplus U^{*}, b_{\mathbb{M}(U, b)}\right)$ whose bilinear form $b_{\mathbb{M}(U, b)}$ is defined by

$$
b_{\mathbb{M}(U, b)}(u+\varphi, v+\psi)=b(u, v)+\varphi(v)+\psi(u) .
$$

It is a regular bilinear form, justifying the terminology "space". By definition, the hyperbolic bilinear space is $\mathbb{H}_{b}(U)=\mathbb{M}(U, 0)$ where 0 is the null form.

Given a finite projective $R$-module $U$, the associated hyperbolic space $\mathbb{H}(U)$ is the quadratic module $\left(U^{*} \oplus U\right.$, hyp) with $\operatorname{hyp}(\varphi \oplus u)=\varphi(u)$. The polar form of the hyperbolic quadratic form hyp is the bilinear form $b_{\mathbb{H}_{b}(U, 0)}$, in particular, hyp is nonsingular. We call $\mathbb{H}(R)$ the hyperbolic plane.

We say a bilinear module $(M, b)$ is metabolic if there exists a bilinear module $\left(U, b_{U}\right)$ such that $(M, b) \cong \mathbb{M}\left(U, b_{U}\right)$. The same terminology will be applied to hyperbolic quadratic spaces. The following facts are for example proven in [Ba, I, §3 and §4].

(a) Let $(M, b)$ be a regular bilinear module and let $U \subset M$ be a totally isotropic complemented submodule. Then there exists a submodule $V \subset M$ such that $U \cap V=0,\left(U \oplus V,\left.b\right|_{U \oplus V}\right)$ is metabolic and hence $M=(U \oplus V) \perp$ $(U \oplus V)^{\perp}$. If $b\left(m_{1}, m_{2}\right)=b_{0}\left(m_{1}, m_{2}\right)+b_{0}\left(m_{2}, m_{1}\right)$ for some in general non-symmetric bilinear form $b_{0}: M \times M \rightarrow R$, one can choose $V$ such that $\left(U \oplus V,\left.b\right|_{U \oplus V}\right)$ is a hyperbolic bilinear space.

(b) (Characterization of metabolic spaces) A regular bilinear module $(M, b)$ is metabolic if and only if one of the following conditions holds:

(i) $M$ contains a totally isotropic complemented submodule $V$ with $V=$ $V^{\perp}$, a so-called Lagrangian,

(ii) $M$ contains a totally isotropic and complemented submodule $U$ satisfying $\operatorname{rank}_{\mathfrak{p}} M=2 \operatorname{rank}_{\mathfrak{p}} U$ for all $\mathfrak{p} \in \operatorname{Spec}(R)$.

In this case $(M, b) \cong \mathbb{M}(U)$ for $U \cong V^{*}$. In particular, $\mathbb{M}(U)$ is free whenever $V$ is free, and any decomposition $V=V_{1} \oplus \cdots \oplus V_{n}$ gives rise to a decomposition $\mathbb{M}(U)=\mathbb{M}\left(U_{1}\right) \perp \cdots \perp \mathbb{M}\left(U_{n}\right)$ with $U_{i} \cong V_{i}^{*}$. 
To see that $M$ is metabolic in case (ii), apply (a) to get $M=(U \oplus V) \perp$ $(U \oplus V)^{\perp}$ where $U \oplus V$ is metabolic and $\operatorname{rank}_{\mathfrak{p}}=2 \operatorname{rank}_{\mathfrak{p}} U=\operatorname{rank}_{\mathfrak{p}} M$, whence $(U \oplus V)^{\perp}=0$. The last claim follows from $V^{*}=V_{1}^{*} \oplus \cdots \oplus V_{n}^{*}$.

In the remainder of this section we consider quadratic spaces $(M, q)$ over $R$ in two settings: (a) $q$ is regular, and (b) $R$ is semilocal. The results in case (a) are proven in [Ba, $\S 3$ and $\S 4]$. Since our techniques in case (b) easily also lead to proofs in the setting of (a), we prove (a) and (b) at the same time. We first recall a folklore result about lifting of subspaces in the semilocal setting. It is straightforward to prove or can be obtained by specializing the Reduction Theorem [Knu, II, (4.6.1)].

1.4. Lemma. Let $R$ be a semilocal ring, let $M$ be a finite projective $R-$ module and let $U \subset M$ be a complemented submodule. For a maximal ideal $\mathfrak{m} \triangleleft R$ we put $\kappa(\mathfrak{m})=R / \mathfrak{m}, M(\mathfrak{m})=M \otimes_{R} \kappa(\mathfrak{m})=M / \mathfrak{m} M$, and analogously for $U(\mathfrak{m})$. We further assume that $r \in \mathbb{N}_{+}$and that for every $\mathfrak{m} \in \operatorname{Specmax}(R)$ there exists an $r$-dimensional subspace $W[\mathfrak{m}] \subset M(\mathfrak{m})$ with $U(\mathfrak{m}) \cap W[\mathfrak{m}]=\{0\}$.

Then there exists a free submodule $W \subset M$ of rank $r$ which satisfies $W(\mathfrak{m})=W[\mathfrak{m}], U \cap W=\{0\}$ and which has the property that $U \oplus W$ is complemented in $M$.

1.5. Proposition. Let $(M, q)$ be a quadratic space over $R$, let $U \subset M$ be a complemented totally isotropic submodule, and assume one of the following.

(a) $q$ is regular, or

(b) $R$ is semilocal.

Then there exists a totally isotropic submodule $V \subset M$ such that $U \cap V=$ $\{0\},\left(U \oplus V, q_{U \oplus V}\right) \cong \mathbb{H}(U)$ and hence $M=(U \oplus V) \oplus(U \oplus V)^{\perp}$.

Proof. (I) (Intermediate step) Suppose there exists a submodule $W \subset M$ satisfying

$$
\begin{aligned}
& U \cap W=\{0\}, \text { and for which the canonical map } \\
& \beta: U \stackrel{\sim}{\longrightarrow} W^{*}, \quad u \mapsto\left(w \mapsto b_{q}(u, w)\right)
\end{aligned}
$$

is an isomorphism of $R$-modules. By [Ba, I, (1.7)], we can choose a not necessarily symmetric bilinear form $b_{0}$ satisfying $b_{0}(m, m)=q(m)$ for all $m \in M$. By (1.5.1), for every $w \in W$ there exists a unique $u_{w} \in U$ such that $b_{q}\left(u_{w}, w^{\prime}\right)=b_{0}\left(w, w^{\prime}\right)$ holds for all $w^{\prime} \in W$. Because of uniqueness of the $u_{w}$, the map $W \rightarrow U, w \mapsto u_{w}$, is $R$-linear. Then $V=\left\{w-u_{w}: w \in W\right\} \cong W$ is a totally isotropic submodule: since $q\left(u_{w}\right)=0$ we have

$$
q\left(w-u_{w}\right)=q(w)-b_{q}\left(w, u_{w}\right)=b_{0}(w, w)-b_{q}\left(u_{w}, w\right)=0 .
$$

Moreover, $U \cap V=0$ and the canonical map $U \stackrel{\sim}{\longrightarrow} V^{*}, u \mapsto\left(v \mapsto b_{q}(u, v)\right)$ is an isomorphism. Hence $\left(U \oplus V,\left.q\right|_{U \oplus V}\right) \cong \mathbb{H}(U)$. Since $\mathbb{H}(U)$ is a regular quadratic module, 1.1(e) applies and yields $M=(U \oplus V) \oplus(U \oplus V)^{\perp}$. 
In the remainder of the proof we will establish the existence of a submodule $W$ satisfying (1.5.1). We point out that (I) applies to the two cases (a) and (b).

(II) (Case (a) in general and case (b) with $R$ a field) Let $U^{\perp}=\{m \in M$ : $\left.b_{q}(m, U)=0\right\}$. We have a well-defined pairing

$$
\alpha: U \times M /\left(U^{\perp}\right) \rightarrow R, \quad(u, \bar{m}) \mapsto b_{q}(u, m)
$$

which is regular: if $\alpha(u, \bar{m})=b_{q}(u, m)=0$ for all $m \in M$, then $u \in$ $\operatorname{rad}\left(b_{q}\right)$. Hence $u=0$ in case (a), while in case (b) we get $u \in \operatorname{rad}(q)$ because $q(u)=0$, so that again $u=0$ follows, using that $\operatorname{rad}(q)=0$ if $R$ is a field. Also, if $\alpha(U, \bar{m})=b_{q}(U, m)=0$, then $m \in U^{\perp}$, and therefore $\bar{m}=0$ follows. We now get that $\alpha$ induces an isomorphism $\alpha^{*}: U \stackrel{\sim}{\longrightarrow}$ $\left(M / U^{\perp}\right)^{*}$. We claim that there exists a submodule $W \subset M$ such that $M=$ $U^{\perp} \oplus W$, and that therefore we have the canonical isomorphism can: $W \stackrel{\sim}{\longrightarrow}$ $M / U^{\perp}$. The existence of $W$ follows in case (a) from [Ba, I, (3.2)(a)], saying that $U^{\perp}$ is complemented in $M$. It is obvious in case (b) with $R$ a field. Denoting by can* $\left(M / U^{\perp}\right)^{*} \stackrel{\sim}{\longrightarrow} W^{*}$ the dual of the isomorphism can, we have $\left(\left(\operatorname{can}^{*} \circ \alpha^{*}\right)(u)\right)(w)=\alpha^{*}(u)(\operatorname{can}(w))=\alpha^{*}(u)(\bar{w})=b_{q}(u, w)=$ $(\beta(u))(w)$, i.e., $W$ satisfies (1.5.1). Indeed, we have $W \cap U=\{0\}$ (since $U \subset U^{\perp}$ and $\left.W \cap U^{\perp}=\{0\}\right)$ and with $\beta=$ can $^{*} \circ \alpha^{*}$. Now (I) finishes the proof in case (a), and in case (b) with $R$ a field. Before we can deal with a semilocal $R$ in case (b) we make a further reduction.

(III) (Reduction to constant rank) Since $U$ is complemented, it is finitely generated projective. By 1.1(i), we can therefore decompose $R=R_{1} \times \cdots \times$ $R_{n}$ and correspondingly

$$
(M, q)=\left(M_{1}, q_{1}\right) \perp \cdots \perp\left(M_{n}, q_{n}\right), \quad U=U_{1} \times \cdots \times U_{n}
$$

such that each $U_{i} \subset M_{i}$ is a complemented submodule of constant rank and a totally isotropic submodule of the $R_{i}$-quadratic space $\left(M_{i}, q_{i}\right)$. If $V_{i} \subset M_{i}, 1 \leq i \leq n$, are submodules as in the claim of the lemma, then $V=$ $V_{1} \times \cdots \times V_{n}$ satisfies the conditions for $(M, q)$. Without loss of generality we can therefore assume that $U$ has constant rank, say rank $r$. It is then free of rank $r$.

(IV) (Case (b) in general) By (III) we can assume that $U$ is free of rank $r$. Using the notation of Lemma 1.4 , we know that $q_{\kappa(\mathfrak{m})}$ is nonsingular by $1.2(\mathrm{~d})$. Thus, by (II), the lemma holds for $\kappa(\mathfrak{m})$. We can therefore choose an $r$-dimensional subspace $W[\mathfrak{m}]$ such that $U(\mathfrak{m}) \cap W[\mathfrak{m}]=\{0\}$ and $U(\mathfrak{m}) \stackrel{\sim}{\longrightarrow} W[\mathfrak{m}]^{*}$ via $b_{\kappa(\mathfrak{m})}$. By Lemma 1.4 , the $W[\mathfrak{m}]$ lift to a submodule $W$ satisfying $U \cap W=\{0\}$ and $W(\mathfrak{m})=W[\mathfrak{m}]$. Moreover, the map $U \longrightarrow W^{*}$, induced by $b_{q}$, is an isomorphism by Nakayama, since it is an isomorphism after passing to each $\kappa(\mathfrak{m})$. Again (I) finishes the proof.

1.6. Corollary (Characterization of hyperbolic spaces). Let $(M, q)$ be a quadratic $R$-space and assume that $q$ is regular or that $R$ is semilocal. Then the following are equivalent: 
(i) $(M, q)$ is hyperbolic;

(ii) $M$ admits a complemented submodule $U$ satisfying $q(U)=0$ and $2 \operatorname{rank}_{\mathfrak{p}} U=\operatorname{rank}_{\mathfrak{p}} M$ for all $\mathfrak{p} \in \operatorname{Spec}(R)$;

(iii) $M$ admits a complemented submodule $U$ satisfying $q(U)=0$ and $U=U^{\perp}$.

In this case $(M, q) \cong \mathbb{H}(U)$.

Proof. (i) $\Longrightarrow$ (ii) being obvious because $\operatorname{rank}_{\mathfrak{p}} U=\operatorname{rank}_{\mathfrak{p}} U^{*}$, let us assume (ii) and prove (iii). By Proposition 1.5, there exists a submodule $V \subset M$ such that $\left(U \oplus V,\left.q\right|_{U \oplus V}\right) \cong \mathbb{H}(U)$ is hyperbolic and $M=(U \oplus V) \oplus(U \oplus V)^{\perp}$. Since $V \cong U^{*}$ as $R$-modules, $\operatorname{rank}_{\mathfrak{p}}(U \oplus V)=\operatorname{rank}_{\mathfrak{p}} M$, whence $U \oplus V=M$ and $U^{\perp}=U \oplus\left(U^{\perp} \cap V\right)$ with $U^{\perp} \cap V \cong\left\{\varphi \in U^{*}: \varphi(U)=0\right\}=\{0\}$. The proof of (iii) $\Longrightarrow$ (i) follows the same pattern.

1.7. Corollary. Let $(M, q)$ and $\left(M^{\prime}, q^{\prime}\right)$ be regular quadratic modules.

(a) $[\mathrm{Ba}, \mathrm{I},(4.7 . \mathrm{i})]$ If $(M, q)$ and $\left(M^{\prime}, q^{\prime}\right)$ are isometric, then the quadratic module $(M, q) \perp\left(M^{\prime},-q^{\prime}\right)$ is hyperbolic: $(M, q) \perp\left(M^{\prime},-q^{\prime}\right) \cong \mathbb{H}(M)$.

(b) Conversely, if $R$ is semilocal and if $(M, q) \perp\left(M^{\prime},-q^{\prime}\right) \cong \mathbb{H}(M)$, then $(M, q) \cong\left(M^{\prime}, q^{\prime}\right)$.

Proof. (a) Let $f:(M, q) \rightarrow\left(M^{\prime}, q^{\prime}\right)$ be an isometry. The quadratic form $q \perp\left(-q^{\prime}\right)$ is regular. The diagonal submodule $U=\{(m, f(m)): m \in M\} \subset$ $M \oplus M^{\prime}$ is complemented by $\{(m, 0): m \in M\}$ and satisfies 1.6(ii). Hence $(M, q) \perp\left(M^{\prime},-q^{\prime}\right) \cong \mathbb{H}(M)$.

(b) By (a), $(M, q) \perp(M,-q) \cong \mathbb{H}(M) \cong(M, q) \perp\left(M^{\prime},-q^{\prime}\right)$. Hence $(M,-q) \cong\left(M^{\prime}-q^{\prime}\right)$ by Witt cancellation $1.2(\mathrm{j})$, which implies our claim.

Corollary 1.7(a) is not true for nonsingular quadratic forms, even over fields. For example, let $(M, q)=\left(F,\langle u\rangle_{q}\right)=\left(M^{\prime}, q^{\prime}\right)$ with $F$ a field of characteristic 2 and $u \in F^{\times}$. Then $(M, q)$ is nonsingular by $1.2(\mathrm{~h})$, but $0 \neq\left(1_{F}, 1_{F}\right) \in \operatorname{rad}(q \perp(-q))$, so that $q \perp(-q)$ is singular, hence in particular not hyperbolic.

1.8. Unimodular and isotropic vectors. Let $M$ be a finite projective $R$-module. For $x \in M$ and $\mathfrak{p} \in \operatorname{Spec}(R)$ we put $x(\mathfrak{p})=x \otimes_{R} 1_{\kappa(p)}$. Recall that $u \in M$ is called unimodular if $u$ satisfies one of the following equivalent conditions, see e.g. [Lo, 0.3]:

(i) $R u$ is complemented and free of rank 1 ,

(ii) there exists $\varphi \in M^{*}$ satisfying $\varphi(u)=1$,

(iii) $u(\mathfrak{p}) \neq 0$ for all $\mathfrak{p} \in \operatorname{Spec}(R)$,

(iv) $u(\mathfrak{m}) \neq 0$ for all maximal $\mathfrak{m} \in \operatorname{Spec}(R)$.

Let $(M, q)$ be a quadratic module. We call $m \in M$ isotropic if $m$ is unimodular and $q(m)=0$. We say $(M, q)$ is isotropic if $M$ contains an isotropic vector. We note some useful facts.

(a) If $m$ is a unimodular (resp. isotropic) vector of $(M, q)$, then $m \otimes 1_{S}$ is a unimodular (resp. isotropic) vector of $(M, q)_{S}$ for any $S \in R$-alg. 
(b) Let $R[X]$ be the polynomial ring over $R$ in the variable $X$ and let $(M, q)$ be a quadratic $R$-module. For $v=v(X) \in M \otimes_{R} R[X]$ define the affine $R$-scheme

$$
Z_{v}=\left\{x \in \mathbb{G}_{a, R}: v(x)=0\right\},
$$

whose $T$-points, $T \in R$-alg, is the set $Z_{v}(T)=\{t \in T: v(t)=0\}$ where $v(t) \in M \otimes_{R} T$ is obtained by substituting $t$ for $X$. Then

$$
Z_{v} \text { empty } \Longrightarrow v \text { unimodular. }
$$

Indeed, if $\mathfrak{p} \in \operatorname{Spec}(R[X])$, then $v(\mathfrak{p})=v\left(X \otimes 1_{\kappa(\mathfrak{p})}\right) \neq 0$ since otherwise $X \otimes 1_{\kappa(\mathfrak{p})} \in Z_{v}(\kappa(\mathfrak{p}))$.

(c) Let $(M, q)$ be a quadratic space over $R$ and assume that $q$ is regular or that $R$ is semilocal. By Proposition 1.5, any isotropic vector embeds into a hyperbolic plane $\mathbb{H}=\mathbb{H}(R)$ and $(M, q)=\mathbb{H} \perp\left(M_{1}, q_{1}\right)$. In particular, $\operatorname{rank}_{\mathfrak{p}} M \geq 2$ holds for every $\mathfrak{p} \in \operatorname{Spec}(R)$.

(d) Let $M$ be a projective $R$-module of constant rank 2 and let $q: M \rightarrow R$ be a nonsingular quadratic form. By 1.2(e), $q$ is nonsingular if and only if $q$ is regular. Hence, by (c), we have the implication " $\Longrightarrow$ " of

$$
(M, q) \text { isotropic } \Longleftrightarrow(M, q) \cong \mathbb{H}(R) .
$$

Thus, in this case $M$ is free of rank 2. The other direction in (1.8.2) holds because in $\mathbb{H}(R)=R \oplus R$ with the hyperbolic form hyp, given by $\operatorname{hyp}\left(r_{1}, r_{2}\right)=r_{1} r_{2}$, the vector $(1,0)$ is isotropic.

\section{SPRINGER's ODD EXTENSION THEOREM}

Let $(M, q)$ be a quadratic module. To simplify notation, we will often abbreviate $q(x)=q_{S}(x)$ for $x \in M_{S}$ if $S$ is clear from the context. We will also say that $q$ is $S$-isotropic if $q_{S}$ is isotropic, cf. 1.8. We recall that a quadratic space $(M, q)$ is a quadratic module with a nonsingular $q$.

2.1. Theorem (Springer's Theorem). Let $R$ be a semilocal ring and let $(M, q)$ be a quadratic space. Let $S$ be a finite $R$-algebra of constant odd degree, which is étale or one-generated. If $q$ is $S$-isotropic, then $q$ is $R$ isotropic.

Remarks. The formulation of Theorem 2.1 captures the essence of our work. It is immediate that the theorem extends to $S \in R$-alg admitting a tower $R=S_{0} \subset S_{1} \subset \cdots \subset S_{n}=S$ for which each $S_{i}$ is a finite $S_{i-1}$-algebra of constant odd degree which is one-generated or étale. The same observation applies to the corollaries stated below. We will use this observation in the proof of case (iii) in Corollary 2.8.

The proof of this theorem will be given in 2.5. Lemma 2.2 proves Springer's Theorem in the case of $\operatorname{rank} M=2$. It involves a much weaker condition than $R$ being semilocal or that $S$ is one-generated or étale. By 1.2(e), a quadratic form on such an $R$-module $M$ is nonsingular if and only if it is regular. 
2.2. Lemma (Rank 2). Let $S \in R$-alg be a finite $R$-algebra of constant odd degree and let $(M, q)$ be a quadratic space of constant rank 2 . Then $(M, q)$ is isotropic if and only if $(M, q)_{S}$ is isotropic.

Proof. It is clear that $(M, q)$ isotropic $\Longrightarrow(M, q)_{S}$ isotropic. Let us therefore assume that $(M, q)_{S}$ is isotropic. Let $A$ be the even part of the Clifford algebra $C=C(q)$ associated with $(M, q)$; we can and will identify $M$ with the odd part of $C$. It is known [Knu, $\mathrm{V},(2.1)]$ that $A$ is a quadratic étale $R$-algebra; let $n_{A}$ be its norm. The multiplication of $C$ makes $M$ a projective right $A$-module of rank 1 satisfying $q(m a)=q(m) n_{A}(a)$ for $m \in M$ and $a \in A$.

Since $q_{S}$ is isotropic, $(M, q)_{S}$ is a hyperbolic $S$-plane by (1.8.2). Hence $C \otimes_{R} S \cong C\left(q_{S}\right)$ is the split quaternion $S$-algebra and $A_{S}$ is the split quadratic étale $S$-algebra. By [Knu, III, (4.1.2)], the automorphism group scheme of the $R$-algebra $A$ is the abelian constant $R$-group scheme $\mathbb{Z} / 2 \mathbb{Z}$. Since $S$ has constant odd degree, we are in the setting of the Example in B.4. Thus already $\left(A, n_{A}\right)$ is split, i.e., $A=R \times R$ and $n_{A}$ is hyperbolic. Let $e_{1}=\left(1_{R}, 0\right)$ and $e_{2}=\left(0,1_{R}\right)$ be the standard idempotents of $A$. Then $M=M e_{1} \times M e_{2}$ is the direct product of projective $R$-modules of rank 1 satisfying $q\left(M e_{i}\right)=q(M) n_{A}\left(e_{i}\right)=0$ for $i=1,2$. Finally, by Corollary 1.6, $(M, q)$ is hyperbolic, and hence in particular isotropic.

Our proof of Springer's Theorem also uses the following Lemma 2.3, a variation of [PR, Prop. 1.1].

2.3. Lemma. Let $k$ be a field, let $(V, q)$ be an isotropic quadratic $k$-space of dimension $r \geq 3$, and let $P=P(X) \in k[X]$ be a monic polynomial of degree $d \geq 1$. Then there exists $v=v(X) \in V \otimes_{k} k[X]$ satisfying the following conditions:

(i) $q(v(X)) \in k[X]$ is a polynomial of degree $2 d-2$, which is divisible by $P$;

(ii) the $k$-scheme $Z_{v(X)}=\left\{x \in \mathbb{G}_{a}: v(x)=0\right\}$ is empty.

In particular, $v(X)$ is unimodular.

Proof. Since $q$ is isotropic, $(V, q)$ contains a hyperbolic plane $\mathbb{H}$ and $(V, q)=$ $\mathbb{H} \perp\left(W,\left.q\right|_{W}\right)$ for $\left(W,\left.q\right|_{W}\right)=\mathbb{H}^{\perp}$, see $1.8(\mathrm{c})$ or [EKM, 7.13]. The quadratic module $\left(W,\left.q\right|_{W}\right)$ contains $w \in W$ with $q(w)=: a \in k^{\times}$. In view of our claims, it is then no harm to replace $V$ by $\mathbb{H} \oplus k a$. Thus $q$ is given by $q(x, y, z)=x y+a z^{2}$, which is nonsingular by $1.2(\mathrm{~g})$ and $1.2(\mathrm{~h})$ (but not regular in characteristic 2).

Since $P$ is monic, the Euclidean division algorithm yields unique polynomials $Q(X) \in k[X]$ of degree $d-2$ and $R(X)$ of degree $\leq d-1$ such that $X^{2 d-2}=P(X) Q(X)+R(X)$. We define $v(X)=\left(-a, R(X), X^{d-1}\right) \in$ $V \otimes k[X]$. Then $q(v(X))=-a R(X)+a X^{2 d-2}=a P(X) Q(X)$. Thus, the condition (i) is fulfilled with $q(v(X))=a P(X) Q(X)$. Since the first component of $v(X)$ is $-a$, the condition (ii) is satisfied too. It implies unimodularity of $v(X)$ by (1.8.1). 
2.4. Consequences of Lemma 2.3. As motivation for step (III) in the proof of Theorem 2.1 below, we discuss some consequences of Lemma 2.3. Step (III) will be more technical, but uses the same ideas. Let us put

$$
S=k[X] /(P), \quad \theta=X+(P) \in S .
$$

Then $S$ is one-generated with $\theta$ as primitive element. The element $v(\theta) \in$ $\left(V \otimes_{k} k[X]\right) \otimes_{k[X]} S=V \otimes_{k} S$ is unimodular since it is obtained from the unimodular $v(X)$ by base change, see $1.8($ a), and it satisfies $q(v(\theta))=0$ by condition (i) of 2.3, i.e., $v(\theta)$ is an isotropic vector in $V \otimes_{k} S$.

Again by condition (i) of 2.3, the exists $u \in k^{\times}$and $Q(X) \in k[X]$ of degree $d-2$ such that $q(v(X))=u P(X) Q(X) \in k[X]$. Let

$$
T=k[X] /(Q), \quad \vartheta=X+(Q) \in T .
$$

Then $T$ is one-generated of degree $d-2$ with primitive element $\vartheta$. The same arguments showing that $v(\theta)$ is an isotropic vector proves that $v(\vartheta) \in V \otimes_{k} T$ is isotropic.

In step (III) of the proof of Theorem 2.1 we will know that $V \otimes_{k} S$ is isotropic and use a refinement of the argument above to conclude that $V \otimes_{k} T$ is isotropic, the point being that $\operatorname{deg} T=\operatorname{deg} S-2$.

2.5. Proof of Theorem 2.1. (I) Reduction to $M$ free of rank $r \geq 3$. Let $R=R_{0} \times \cdots \times R_{n}$ and $(M, q)=\left(M_{0}, q_{0}\right) \perp \cdots \perp\left(M_{n}, q_{n}\right)$ be the rank decomposition of $(M, q)$ as in 1.2(i). Thus, $M_{i}$ is a projective $R_{i}$-module of rank $i$ and each $q_{i}: M_{i} \rightarrow R_{i}$ is a nonsingular quadratic form. The $R$ algebra $S$ decomposes correspondingly, $S=S_{0} \times \cdots \times S_{n}$ where each $S_{i}$ is a finite $R_{i}$-algebra of degree $d=\operatorname{deg} S$. We have

$$
M \otimes_{R} S \cong\left(M_{0} \otimes_{R_{0}} S_{0}\right) \times \cdots \times\left(M_{n} \otimes_{R_{n}} S_{n}\right)
$$

with each $M_{i} \otimes_{R_{i}} S_{i}$ being projective of rank $i$ as $S_{i}$-module. Since $q_{S}$ is isotropic, so is every $\left(q_{i}\right)_{S_{i}}$. By $1.8(\mathrm{c}), M_{i} \otimes_{R_{i}} S_{i}=0$ for $i=0,1$. Since in both cases ( $S$ one-generated or $S$ étale) the $R_{i}$-modules $S_{i}$ are faithfully flat, we get $M_{0}=0=M_{1}$ and $R_{0}=0=R_{1}$.

In the decomposition $R=R_{2} \times \cdots \times R_{n}$, each $R_{i}$ is a semilocal ring. We have already observed that $(M, q)_{S}$ is isotropic if and only if every $M_{i} \otimes_{R_{i}} S_{i}$ is isotropic. Since the analogous fact holds for $(M, q)$, it suffices to prove that every $\left(M_{i}, q_{i}\right)$ is isotropic. Thus, without loss of generality, we can assume that $M$ has constant rank $r \geq 2$. The case $r=2$ has been dealt with in Lemma 2.2. We can therefore assume that $M$ has rank $r \geq 3$. Since $R$ is semilocal, this implies that $M$ is free of rank $r$.

(II) $R=k$ is a field and $S$ is one-generated. In this case $S \cong k[X] / P$ for some monic $P \in k[X]$. Let $P=P_{1}^{e_{1}} \cdots P_{n}^{e_{n}}$ be the prime factor decomposition of $P$ in $k[X]$, and put $L_{i}=k[X] / P_{i}$. Since $d=\operatorname{dim}_{k} S=\sum_{i} e_{i}\left[L_{i}: k\right]$ is odd, one of the $\left[L_{i}: k\right]$ is odd. Then $L=L_{i} \in S$-alg and thus $q_{L}=\left(q_{S}\right) \otimes_{S} L$ is isotropic. Since $L / k$ has odd degree, the classical Springer Odd Degree Extension Theorem [EKM, Cor. 18.5] says that $q$ is isotropic. 
(III) $R$ semilocal and $S$ is one-generated. As before, let $S=R[X] /(P)$ where $P \in R[X]$ is a monic polynomial of degree $d$. We denote by $\kappa_{1}, \ldots, \kappa_{c}$ the residue fields of $R$. For each $i, 1 \leq i \leq c$, the $\kappa_{i}$-algebra $S_{\kappa_{i}}=S \otimes_{R} \kappa_{i}$ is one-generated, namely $S_{\kappa_{i}}=\kappa_{i}[X] / P_{\kappa_{i}}$ for $P_{\kappa_{i}}=P \otimes_{R} 1_{\kappa_{i}}$, and of odd degree $d$. Since $q$ is $S$-isotropic, it is also $S \otimes_{R} \kappa_{i}$-isotropic. The case (II) then shows that $q_{\kappa_{i}}$ is isotropic. Now Lemma 2.3 provides unimodular elements $v_{i}(X) \in\left(M \otimes_{R} \kappa_{i}\right) \otimes_{\kappa_{i}} \kappa_{i}[X]=M \otimes_{R} \kappa_{i}[X]$ such that $q\left(v_{i}(X)\right)$ is the product of a unit in $\kappa_{i}^{\times}$and a monic polynomial of degree $2 d-2$ which is divisible by $P_{\kappa_{i}}$ and has the property that the $\kappa_{i}$-scheme

$$
Z_{v_{i}}=\left\{x \in \mathbb{G}_{a, \kappa_{i}}: v_{i}(x)=0\right\} \text { is empty. }
$$

Let $\theta=X+(P) \in S$ and denote by $\theta_{i}$ its image in $S_{\kappa_{i}}$. Then $v_{i}\left(\theta_{i}\right)$ is obtained from the unimodular $v_{i}(X)$ by base change and is therefore unimodular. It also satisfies $q\left(v_{i}\left(\theta_{i}\right)\right)=0$ since $q\left(v_{i}(X)\right)$ is divisible by $P_{\kappa_{i}}$. In other words, $v_{i}\left(\theta_{i}\right)$ is an $S_{\kappa_{i}}$-isotropic vector.

According to Corollary A.6 of the appendix, the $v_{i}\left(\theta_{i}\right)^{\prime} s \in M \otimes_{R} S_{\kappa_{i}}$ lift to an isotropic $v \in M \otimes_{R} S$. We decompose $v=m_{0}+m_{1} \theta+\cdots+m_{d-1} \theta^{d-1}$ where the $m_{j}$ 's belong to $M$, and define $v(X)=m_{0}+m_{1} X+\cdots+m_{d-1} X^{d-1} \in$ $M \otimes_{R} R[X]$. By construction $q(v(X)) \in P(X) R[X]$ is a polynomial of degree $\leq 2 d-2$. Since the specialization to each $\kappa_{i}[X]$ is of degree $2 d-2$, it follows that $q(v(X))$ is the product of a unit $u \in R^{\times}$and a monic polynomial of degree $2 d-2$. Summarizing, we have that $q(v(X))=u P(X) Q(X)$ with $Q(X)$ monic of degree $d-2$ and $u \in R^{\times}$.

Since $Z_{v_{i}}=\left\{t \in \mathbb{G}_{a, \kappa_{i}}: v_{i}(t)=0\right\}$ is empty for each $i$, it follows that $Z_{v}=\left\{x \in \mathbb{G}_{a, R}: v(x)=0\right\}$ is empty too. We define $T=R[X] / Q(X)$. Then $w=v(X)$ modulo $Q$ is an isotropic vector of $M_{T}$. Thus, $q$ is $T$-isotropic with $T$ one-generated of degree $d-2$. We continue the induction until we reach $d-2=1$ and can conclude that $M$ is isotropic.

(IV) $S$ is étale. According to [BFP, Prop. 7.3] there exists a finite étale $R$-algebra $T$ of constant odd degree such that $S \otimes_{R} T$ is one-generated as $R$-algebra. The paper $[\mathrm{BFP}]$ assumes throughout that $2 \in R^{\times}$, but the proof of the quoted proposition works for arbitrary $R$.

Since $q$ is $S$-isotropic, it is a fortiori $S \otimes_{R} T$-isotropic. Since $S \otimes_{R} T$ has constant odd degree, the preceding case (III) shows that $q$ is isotropic.

As in the case of fields, see e.g. [Sch, II], Theorem 2.1 has a number of consequences worth stating. First, by 1.8(c), Springer's Theorem says: if $(M, q)_{S}$ contains a hyperbolic plane $\mathbb{H}$, then so does $(M, q)$. Corollary 2.6 says that this is true for arbitrary hyperbolic spaces. We say a quadratic module $(M, q)$ contains a quadratic module $\left(M_{1}, q_{1}\right)$ if there exists a complemented submodule $N \subset M$ such that $\left(M_{1}, q_{1}\right) \cong\left(N,\left.q\right|_{N}\right)$. In this case, we usually identify $\left(M_{1}, q_{1}\right)=\left(N,\left.q\right|_{N}\right)$. We recall that if $\left(M_{1}, q_{1}\right)$ is regular, e.g., a hyperbolic space, then $(M, q)=\left(M_{1}, q_{1}\right) \perp\left(M_{1}, q_{1}\right)^{\perp}$ by 1.1(e). 
2.6. Corollary. Let $R, S$ and $(M, q)$ be as in Theorem 2.1. If $(M, q)_{S}$ contains a hyperbolic space $\mathbb{H}\left(N^{\prime}\right)$ with $N^{\prime}$ projective of constant rank $r$, then $(M, q)$ contains a hyperbolic space $\mathbb{H}(N)$ with $N$ projective of rank $r$.

2.7. Corollary. Let $R, S$ and $(M, q)$ be as in Theorem 2.1, let $\left(M_{1}, q_{1}\right)$ be a regular quadratic $R$-module such that $\left(M_{1}, q_{1}\right)_{S}$ is contained in $(M, q)_{S}$. Then $\left(M_{1}, q_{1}\right)$ is contained in $(M, q)$.

Proof. We apply the rank decomposition 1.2(i) to $\left(M_{1}, q_{1}\right)$ and can then without loss of generality assume that $\left(M_{1}, q_{1}\right)$ has constant rank. Since $\left(M_{1}, q_{1}\right)_{S}$ is regular, the nonsingular quadratic $S$-space $(M, q)_{S}$ decomposes,

$$
(M, q)_{S} \cong\left(M_{1}, q_{1}\right)_{S} \perp\left(M_{2}^{\prime}, q_{2}^{\prime}\right)
$$

where $\left(M_{2}^{\prime}, q_{2}^{\prime}\right)$ is a nonsingular $S$-space by $1.2(\mathrm{~g})$. By Corollary 1.7 , the quadratic $S$-module $q_{S} \perp\left(-q_{1}\right)_{S}$ contains a hyperbolic $S$-space isometric to $\mathbb{H}\left(M_{1, S}\right)$. Hence by Corollary 2.6, there exists a nonsingular quadratic $R$-module $\left(M_{2}, q_{2}\right)$ such that

$$
q \perp\left(-q_{1}\right) \cong \mathbb{H}\left(M_{1}\right) \perp q_{2} \cong q_{1} \perp\left(-q_{1}\right) \perp q_{2} .
$$

Canceling $-q_{1}$ by Witt cancellation $1.2(\mathrm{j})$, yields the result.

For easier reference, we explicitly state the case $\left(M_{1}, q\right)_{S} \cong\left(M_{1}, q_{1}\right)_{S}$ in the following Corollary 2.8. The case (iii) is obtained by writing $S / R$ as a finite tower of odd one-generated $(=$ simple) field extensions.

2.8. Corollary. Let $R$ be a semilocal ring, let $q$ and $q^{\prime}$ be regular $R$-quadratic forms, and let $S \in R$-alg be a finite $R$-algebra of constant odd degree which satisfies one of the following conditions,

(i) $S$ is a one-generated $R$-algebra, or

(ii) $S$ is an étale $R$-algebra, or

(iii) $R$ is a field and $S / R$ is a field extension.

Then

$$
q_{S} \cong q_{S}^{\prime} \Longleftrightarrow q \cong q^{\prime} .
$$

In Corollary 2.9, $\widehat{\mathrm{W}}_{q}(R)$ and $\mathrm{W}_{q}(R)$ denote the Witt-Grothendieck ring and Witt ring of regular quadratic $R$-modules respectively, as for example defined in $[\mathrm{Ba}, \mathrm{I}, \S 2, \S 4]$. These are commutative associative rings without a multiplicative identity if $2 \notin R^{\times}$. The proof of 2.9 is standard, using Corollary 2.7.

2.9. Corollary. Let $R$ be a semilocal ring and let $S \in R$-alg be a finite $R$-algebra of constant odd degree, which is one-generated or étale. Then the maps

$$
\widehat{\mathrm{W}}_{q}(R) \longrightarrow \widehat{\mathrm{W}}_{q}(S) \quad \text { and } \quad \mathrm{W}_{q}(R) \longrightarrow \mathrm{W}_{q}(S),
$$

induced by $[q] \mapsto\left[q_{S}\right]$, are monomorphisms. 
Corollary 2.9 was established in [Ba, V, Thm.6.9] for Frobenius extensions, based on a detailed study of torsion in the kernels of the maps in (2.9.1) and in this way avoiding Springer's Theorem, which is not proven in $[\mathrm{Ba}]$.

2.10. Set of values. By definition, the set of values $D(q)$ of a quadratic module $(M, q)$ is

$$
\mathrm{D}(q)=q(M) \cap R^{\times} .
$$

We will use the following elementary facts.

(a) The set of values is stable under base change: if $S \in R$-alg, then $\mathrm{D}(q) \otimes 1_{S} \subset \mathrm{D}\left(q_{S}\right)$.

(b) If $q$ contains a hyperbolic plane, then $\mathrm{D}(q)=R^{\times}$. Recall from $1.8(\mathrm{c})$ that $q$ contains a hyperbolic plane whenever $(M, q)$ is an isotropic quadratic space and $R$ is semilocal or $q$ is regular.

(c) (Direct products) Let $R=R_{1} \times R_{2}$ be a direct product. By 1.2(i), the quadratic module $(M, q)$ uniquely decomposes as $(M, q)=\left(M_{1}, q_{1}\right) \times$ $\left(M_{2}, q_{2}\right)$ where $\left(M_{i}, q_{i}\right), i=1,2$, is a quadratic $R_{i}$-module, which is nonsingular if $(M, q)$ is so. We have $\mathrm{D}(q)=\mathrm{D}\left(q_{1}\right) \times \mathrm{D}\left(q_{2}\right)$. An $S \in R$-alg which is projective of rank $d \in \mathbb{N}_{+}$uniquely decomposes as $S=S_{1} \times S_{2}$ where each $S_{i}$ is a projective $R_{i}$-module of rank $d$. In view of 1.2(i), this shows that the determination of $\mathrm{D}(q)$ can often be reduced to that of $\mathrm{D}(q)$ where $(M, q)$ has constant rank.

(d) Let $(M, q)=(R,\langle u\rangle)$ with $u \in R^{\times}$. Then $\mathrm{D}(q)=u R^{\times 2}$. For any $S \in R$-alg which is projective of constant odd rank $d$ we have

$$
a \otimes 1_{S} \in \mathrm{D}\left(q_{S}\right) \quad \Longleftrightarrow \quad a \in \mathrm{D}(q) .
$$

By (a) we only need to prove " $\Longrightarrow$ ". We have $\mathrm{D}\left(q_{S}\right)=\left(u \otimes 1_{S}\right) S^{\times 2}$, and if $a \otimes 1_{S}=\left(u \otimes 1_{S}\right) s^{2}$ for some $s \in S$, then $a^{d}=\mathrm{N}_{S / R}\left(a \otimes 1_{S}\right)=u^{d} \mathrm{~N}_{S / R}(s)^{2}$. Since $a^{d} \in a R^{\times 2}$ and $u^{d} \in u R^{\times 2}$, we get $a \in u R^{\times 2}=\mathrm{D}(q)$.

In the following Corollary 2.11 we will prove (2.10.1) for more general nonsingular forms.

2.11. Corollary. Let $R$ be a semilocal ring, let $a \in R^{\times}$and let $(M, q)$ be a quadratic space for which $q^{\prime}=q \perp\langle-a\rangle$ is nonsingular, cf. 1.2(g). Furthermore, let $S \in R$-alg be a finite $R$-algebra of constant odd degree which is étale or one-generated. Then

$$
a \otimes 1_{S} \in \mathrm{D}\left(q_{S}\right) \quad \Longleftrightarrow \quad a \in \mathrm{D}(q) .
$$

This is a well-known result in case $R$ is a field of characteristic $\neq 2$ and $S / R$ is an extension field, see for example [Lam, VII, Cor. 2.9]. In this case, no assumption on $q^{\prime}$ is necessary.

Proof. We will of course only prove " $\Longrightarrow$ ". By 2.10(c) and 2.10(d) we can assume that $M$ has constant rank $\geq 2$. The assumption implies that $q_{S}^{\prime}$ contains an isotropic vector $(x, 1)$ for some $x \in M_{S}$. Hence, by Theorem 2.1 
for $q^{\prime}$, we get that $q^{\prime}$ is $R$-isotropic, i.e., there exists $m \in M$ and $r \in R$ such that $(m, r) \in M \oplus R$ is unimodular and satisfies $q(m)=a r^{2}$. At this point, two cases are clear:

(i) $r=0$ : Then $m \in M$ is an isotropic vector of $q$. Hence, by $1.8(\mathrm{c})$, $(M, q)$ contains a hyperbolic plane and then we are done by $2.10(\mathrm{~b})$.

(ii) $r \in R^{\times}$: Then $a=q\left(r^{-1} m\right)$ and we are again done.

In particular, (2.11.1) holds in case $R$ is a field. For general $R$ we will choose an isotropic vector of $q^{\prime}$ more carefully.

Let $\mathfrak{m} \triangleleft R$ be a maximal ideal of $R$ with residue field $\kappa=R / \mathfrak{m}$, and put $S \otimes_{R} \kappa=S / \mathfrak{m} S$. We thus have extensions

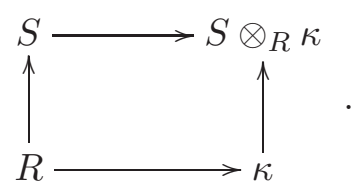

Since nonsingularity is inherited by extensions and since $\left(S \otimes_{R} \kappa\right) / \kappa$ has odd degree and is étale or one-generated, the field case applies and yields the existence of $m_{\kappa} \in M \otimes_{R} \kappa$ satisfying $q_{\kappa}\left(m_{\kappa}\right)=a \otimes 1_{\kappa}$. In this way we obtain a family $\left(v_{\mathfrak{m}}\right)_{\mathfrak{m} \in \operatorname{Specmax}(R)}$ of isotropic vectors $v_{\mathfrak{m}}=\left(m_{\kappa}, 1_{\kappa}\right) \in(M \oplus R)_{\kappa}$. By Corollary A.6, we then get an isotropic element $v=(x, r) \in M \oplus R$ that lifts the $v_{\mathfrak{m}}$ 's. In particular, $r_{\kappa}=1_{\kappa}$ for every residue field $\kappa$ of $R$. This implies $r \in R^{\times}$. So we are done by (ii) above.

\section{Appendix A. Lifting isotropic ElEments}

The goal of this appendix is Corollary A.6, which gives a criterion for lifting isotropic elements from localizations. We obtain this as a consequence of a surjectivity result for localizations of quadrics (Proposition A.5), which in turn is a special case of Demazure's fundamental Conjugacy Theorem, proven in [SGA3, XXVI] and re-stated below.

If $C \in R$-alg and $\mathfrak{m} \triangleleft R$ is a maximal ideal of $R$ we abbreviate $C / \mathfrak{m}:=$ $C / \mathfrak{m} C$. We use the terms reductive (semisimple) group scheme and parabolic subgroup scheme as defined in [SGA3, Tome III], but refer to a group scheme over $\operatorname{Spec}(R)$ as an $R$-group scheme. We furthermore use the setting and the results of [SGA3, XXVI.3] for schemes of parabolic subgroup schemes.

A.1. Theorem (Demazure's Conjugacy Theorem). Let $R$ be a semilocal ring and let $\mathbf{G}$ be a reductive $R$-group scheme. Denote by $\operatorname{Dyn}(\mathbf{G})$ its Dynkin $R$-scheme (which is finite étale) and by $\operatorname{Of}(\operatorname{Dyn}(\mathbf{G}))$ the $R$-scheme of clopen subsets of $\operatorname{Dyn}(\mathbf{G})$. Let $t \in \operatorname{Of}(\operatorname{Dyn}(\mathbf{G}))(R)$ be a type of parabolic subgroups and denote by $\mathbf{X}=\operatorname{Par}(\mathbf{G})_{t}$ the $R$-scheme of parabolic subgroups of type $t$. Then the following hold.

(a) $\mathbf{G}(R)$ acts transitively on $\mathbf{X}(R)$. 
(b) If $S$ is a finite $R$-algebra such that $\mathbf{X}(S) \neq \emptyset$, the map

$$
\mathbf{X}(S) \longrightarrow \prod_{\mathfrak{m} \in \operatorname{Specmax}(S)} \mathbf{X}(S / \mathfrak{m})
$$

is onto.

Proof. (a) If $\mathbf{X}(R)=\emptyset$, the statement is obvious. We can thus assume that $\mathbf{X}(R) \neq \emptyset$ and pick a point $x \in \mathbf{X}(R)$; this corresponds to an $R$-parabolic subgroup $\mathbf{P}$ of $\mathbf{G}$ of type $t$. According to [SGA3, XXVI.4.3.5], $\mathbf{G}$ admits a parabolic subgroup $\mathbf{P}^{\prime}$ opposite to $\mathbf{P}$. Corollary 5.2. of loc. cit. then says in particular that $\mathbf{X}(R)=\mathbf{G}(R) / \mathbf{P}(R)$. Thus $\mathbf{G}(R)$ acts transitively on $\mathbf{X}(R)$.

(b) Recall that $S$ is semilocal, for example by [Knu, VI, (1.1.1)]. Our assumption is that $\mathbf{G}_{S}$ admits an $S$-parabolic subgroup scheme $\mathbf{Q}$ of type $t$. As noted in (a), it admits an opposite parabolic $S$-subgroup $\mathbf{Q}^{\prime}$. According to $\left[\right.$ SGA3, XXVI.5.2], the product $\operatorname{rad}_{u}(\mathbf{Q})(S) \times \operatorname{rad}_{u}\left(\mathbf{Q}^{\prime}\right)(S) \rightarrow \mathbf{X}(S)$ is surjective (here $\operatorname{rad}_{u}($.$) denotes the unipotent radical). Applying this for$ the semilocal ring $S$ as well as for the semilocal $\operatorname{ring} S / \mathfrak{m}, \mathfrak{m} \in \operatorname{Specmax}(R)$, shows that the horizontal maps in the commutative diagram below are surjective:

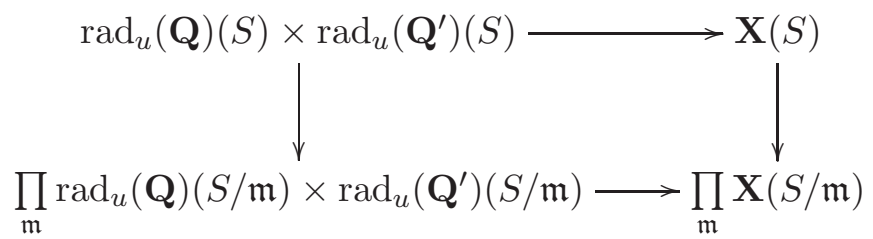

Since $\prod_{\mathfrak{m}} S / \mathfrak{m} \cong \prod_{\mathfrak{m}} S \otimes_{R}(R / \mathfrak{m}) \cong S \otimes_{R}(R / \operatorname{Jac}(R)) \cong S / \operatorname{Jac}(R) S$, where $\operatorname{Jac}(R)$ denotes the Jacobson radical of $R$, the map $S \rightarrow \prod_{\mathfrak{m}} S / \mathfrak{m}$ is onto. On the other hand, the $S$-scheme $\operatorname{rad}_{u}(\mathbf{Q})\left(\right.$ respectively $\left.\operatorname{rad}_{u}\left(\mathbf{Q}^{\prime}\right)\right)$ is isomorphic to a vector $S$-group scheme [SGA3, XXVI.2.5], so that the left vertical map is onto. Hence, by a simple diagram chase the right vertical map is onto too.

A.2. Corollary. We use the notation of A.1, except that $R$ need not be semilocal, but can be arbitrary. In particular, $\mathbf{G}$ is a reductive $R$-group scheme and $x, y$ are parabolic subgroups in $\mathbf{X}(R)$.

Then there exist $f_{1}, \ldots, f_{n} \in R$ satisfying $f_{1}+\cdots+f_{n}=1$ and $y_{R_{f_{i}}} \in$ $\mathbf{G}\left(R_{f_{i}}\right) \cdot x_{R_{f_{i}}}$ for $i=1, . ., n$. In other words, $x$ and $y$ are locally $\mathbf{G}$-conjugated for the Zariski topology on $R$.

Proof. The group $\mathbf{G}$ is an $R$-group of type (RR) by [SGA3, XXII.5.1.3] and the points $x, y$ of $\mathbf{X}(R)$ are parabolic subgroups, hence subgroups of type (R) by 5.2 .3 of loc. cit.. It then follows from Theorem 5.3 .9 of loc. cit. that the strict transporter $\mathbf{T}$, defined by

$$
\mathbf{T}(S)=\left\{g \in \mathbf{G}(S): g \cdot x_{S}=y_{S}\right\} \quad(S \in R \text {-alg }),
$$


is a finitely presented affine $R$-scheme (among other properties). Since $\mathbf{T}\left(R_{\mathfrak{m}}\right) \neq \emptyset$ for any maximal $\mathfrak{m} \in \operatorname{Spec}(R)$ by A.1(a), the claim follows from Lemma A.3 below.

A.3. Lemma. Let $R$ be arbitrary and let $\mathbf{T}$ be an $R$-scheme which is locally of finite presentation. If $\mathbf{T}\left(R_{\mathfrak{m}}\right) \neq \emptyset$ for all maximal $\mathfrak{m} \in \operatorname{Spec}(R)$, then there exists a Zariski cover $\left(f_{1}, \ldots, f_{n}\right)$ of $R$ for which $\mathbf{T}\left(R_{f_{i}}\right) \neq \emptyset$ for all $i$, $1 \leq i \leq n$.

Proof. Fix a maximal $\mathfrak{m} \in \operatorname{Spec}(R)$. Then $R_{\mathfrak{m}}=\underline{\lim }_{f \notin \mathfrak{m}} R_{f}$, hence $\operatorname{Spec}(R)=$ $\varliminf_{f \notin \mathfrak{m}} \operatorname{Spec}\left(R_{f}\right)$ and so $\mathbf{T}\left(R_{\mathfrak{m}}\right)=\underline{\lim }_{f \notin \mathfrak{m}} \mathbf{T}\left(R_{f}\right)$, according to [St, Tag 01ZC]. It follows that there exists $f_{\mathfrak{m}} \in R \backslash \mathfrak{m}$ such that $\mathbf{T}\left(R_{f_{\mathfrak{m}}}\right) \neq \emptyset$. The $f_{\mathfrak{m}}$ 's for $\mathfrak{m}$ running over the maximal ideals of $R$ generate $R$ as ideal. Hence, there exist finitely many maximal ideals $\mathfrak{m}_{1}, \ldots, \mathfrak{m}_{n}$ such that $R=$ $R f_{\mathfrak{m}_{1}}+\cdots+R f_{\mathfrak{m}_{n}}$.

A.4. An important special case of Theorem A.1 and Corollary A.2 is that of quadrics elaborated below. Let us explain some notation. We let $(M, q)$ be a quadratic space with $M$ of constant rank $n$. The associated special orthogonal group scheme $\mathbf{G}=\mathbf{S O}(q)$ is defined in [Co1, C.2.10]. It is a semisimple $R$-group scheme of type $\mathrm{A}_{1}$ for $n=3$, of type $\mathrm{B}_{(n-1) / 2}$ for odd $n \geq 5$ and of type $\mathrm{D}_{n / 2}$ for even $n \geq 4$. We use Bourbaki's enumeration of the corresponding Dynkin diagrams:

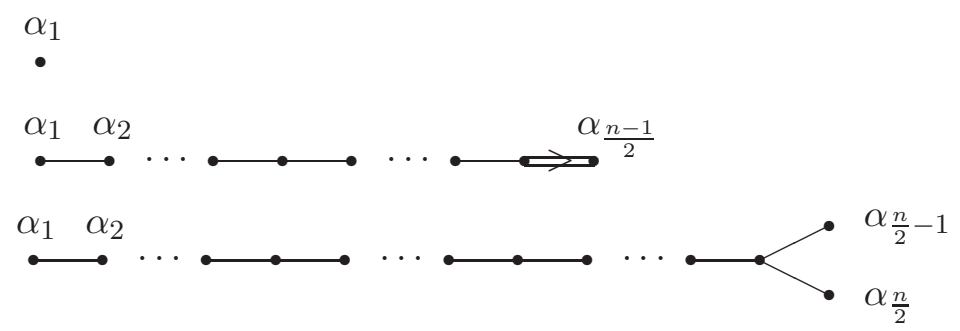

We also use the projective space $\mathbb{P}\left(M^{\vee}\right)$ with Grothendieck's convention, i.e., $\mathbb{P}\left(M^{\vee}\right)(S), S \in R$-alg, corresponds to the complemented submodules $D$ of $M_{S}$ which are locally free of rank 1 . The quadric $\mathbf{Q}$ defined by $q=0$ consists of those $D$ in $\mathbb{P}\left(M^{\vee}\right)(S)$ with $q(D)=0$. For $D \in \mathbf{Q}(R)$ we let $\mathbf{P}$ be the $R$-subgroup scheme of $\mathbf{G}$ which stabilizes $D$. Finally, we remind the reader of our abbreviation $S / \mathfrak{m}=S / \mathfrak{m} S$ for $S \in R$-alg and $\mathfrak{m} \in \operatorname{Specmax}(R)$.

A.5. Proposition. We use the notation of A.4. Then the following hold.

(a) (i) $\mathbf{P}$ is a parabolic $R$-subgroup of $\mathbf{G}$.

(ii) The orbit map $\mathbf{G} \rightarrow \mathbb{P}\left(M^{\vee}\right), g \rightarrow g \cdot[D]$, induces an isomorphism $\mathbf{G} / \mathbf{P} \stackrel{\sim}{\longrightarrow} \mathbf{Q}$ of $R$-schemes.

(iii) $\mathbf{Q}$ is $\mathbf{G}$-isomorphic to $\operatorname{Par}(\mathbf{G})_{t_{1}}$ where the type $t_{1}$ is constant and of value $\operatorname{Dyn}(G)(R) \backslash\left\{\alpha_{1}\right\}_{R}$ with the enumeration of the respective Dynkin diagrams displayed above. 
(b) Let $R$ be semilocal. Then

(i) $\mathbf{G}(R)$ acts transitively on $\mathbf{Q}(R)$, and

(ii) if $S$ is a finite $R$-algebra such that $\mathbf{Q}(S) \neq \emptyset$, then

$$
\mathbf{Q}(S) \longrightarrow \prod_{\mathfrak{m} \in \operatorname{Specmax}(R)} \mathbf{Q}(S / \mathfrak{m})
$$

is onto.

Proof. We prove only the even rank case, the odd case being analogous.

(a) We observe that the claims hold over a field [Co2, Th. 3.9.(i)] and also that they are local for the flat topology. According to [Co1, Lemma C.2.1] or [Knu, IV, (3.2.1)], we may assume that $(M, q)$ is the standard hyperbolic quadratic $R$-form over $R^{2 n}(n \geq 2)$, defined by $q\left(x_{1}, \ldots, x_{n}, y_{1}, \ldots, y_{n}\right)=$ $x_{1} y_{1}+\cdots+x_{n} y_{n}$.

We first consider the case $D=R p$ where $p=(1,0, \ldots, 0,0, \ldots, 0)$, and denote by $\mathbf{P}_{p}$ the corresponding subgroup scheme. This permits us to assume $R=\mathbb{Z}$. Then $\mathbf{P}_{p}$ is an affine finitely presented $\mathbb{Z}$-group scheme whose algebraically closed fibers are smooth connected and of dimension $2 n-2$. By [AG, Lemma B.1], $\mathbf{P}_{p}$ is smooth. Since the geometric fibers of $\mathbf{P}_{p}$ are parabolic subgroups, $\mathbf{P}_{p}$ is parabolic too. The induced orbit map $f: \mathbf{G} / \mathbf{P}_{p} \rightarrow \mathbf{Q}$ is a monomorphism. The field case ensures that this is a fiberwise isomorphism. Since $\mathbf{G} / \mathbf{P}_{p}$ is flat and of finite presentation, the fiberwise isomorphism criterion [EGA, $\mathrm{IV}_{4}, 17.9 .5$ ] enables us to conclude that $f$ is an isomorphism. It follows that $\mathbf{Q}$ is homogeneous under $\mathbf{G}$. Thus (i) and (ii) hold for the special $p$.

Let us now deal with the general case. Since G-homogeneity is a local property with respect to the flat topology, $\mathbf{Q}$ is homogeneous under $\mathbf{G}$. The case of a general $D$ then follows from the observation that $D$ is locally $\mathbf{G}$ conjugated to $R p$ in the Zariski topology by applying Corollary A.2 to Q. This proves (i) and (ii) in general.

(iii) We first deal with the special $p$ above. We have an isomorphism $\mathbf{G} / \mathbf{P} \cong \mathbf{Q}$. On the other hand, according to [SGA3, XXVI.3.6], we have a G-isomorphism $\mathbf{G} / \mathbf{P} \stackrel{\sim}{\longrightarrow} \operatorname{Par}(\mathbf{G})_{t(\mathbf{P})}$, hence an $\mathbf{G}$-isomorphism $\mathbf{Q} \stackrel{\sim}{\longrightarrow}$ $\operatorname{Par}(\mathbf{G})_{t(\mathbf{P})}$. This isomorphism applies a point $x \in \mathbf{Q}(R)$ to the stabilizer $\mathbf{G}_{x}$, so is a canonical isomorphism. Here $t(\mathbf{P}) \in \operatorname{Of}(\operatorname{Dyn}(\mathbf{G}))(R)$ is the type of $\mathbf{P}$. Checking that it is $t_{1}$, reduces to the field case which is [Co2, Lemma $3.12]$.

For the general case, let $S$ be a flat cover of $R$ such that $\mathbf{Q}(S) \neq \emptyset$. We have an isomorphism $\mathbf{Q}_{S} \stackrel{\sim}{\longrightarrow} \operatorname{Par}(G)_{t_{1}, S}$ which is canonical and $\mathbf{G}_{S^{-}}$ equivariant. By faithfully flat descent, it descends to a $\mathbf{G}$-equivariant isomorphism $\mathbf{Q} \stackrel{\sim}{\longrightarrow} \operatorname{Par}(\mathbf{G})_{t_{1}}$.

(b) follows from Theorem A.1 applied to $\mathbf{Q} \cong \operatorname{Par}(\mathbf{G})_{t_{1}}$.

A.6. Corollary. We assume that $R$ is a semilocal ring. Let $(M, q)$ be a quadratic space of constant rank $\geq 3$, let $S$ be a finite $R$-algebra such that 
$q_{S}$ is isotropic, and let $\left(v_{\mathfrak{m}}\right)_{\mathfrak{m} \in \operatorname{Specmax}(R)}$ be a family of isotropic elements $v_{\mathfrak{m}} \in M \otimes_{R} S / \mathfrak{m}$. Then there exists an isotropic $v \in M \otimes_{R} S$ that lifts the $v_{\mathfrak{m}}$ 's.

Proof. Let $\mathbf{Q} \subset \mathbb{P}\left(M^{\vee}\right)$ be the projective quadric associated with $q$ in Proposition A.5. Our assumption is that $\mathbf{Q}(S) \neq \emptyset$.

Each $S / \mathfrak{m}$-module $(S / \mathfrak{m}) \cdot v_{\mathfrak{m}}$ is a complemented submodule of $M \otimes_{R} S / \mathfrak{m}$ which is free of rank one, so defines a point $x_{\mathfrak{m}} \in \mathbf{Q}(S / \mathfrak{m})$. Proposition A.5(b)(ii) provides an element $x \in \mathbf{Q}(S)$ which lifts the $x_{\mathfrak{m}}$ 's. Since $S$ is semilocal, $x$ is represented by an $S$-module $D$ which is a complemented submodule of $M \otimes_{R} S$ of rank one and satisfies $q(D)=0$. We write $D=S v$ where $v$ is an $S$-unimodular element of $M \otimes_{R} S$ satisfying $q(v)=0$. Since $S^{\times} \rightarrow \prod_{\mathfrak{m}}(S / \mathfrak{m})^{\times}$is onto, we can modify $v$ by an unit of $S$ to ensure that $v$ lifts the $v_{\mathfrak{m}}$ 's.

\section{Appendix B. Trace FOR TORSORS}

B.1. Weil restriction ([BLR, 7.6], [CGP, A.5], [DG, I, §1, 6.6]). Let $S \in$ $R$-alg. Given an $S$-functor $Y^{\prime}$, the Weil restriction of $Y^{\prime}$ is the $R$-functor $\mathrm{R}_{S / R}\left(Y^{\prime}\right)$ defined by

$$
\mathrm{R}_{S / R}\left(Y^{\prime}\right)(A)=Y^{\prime}\left(A \otimes_{R} S\right), \quad(A \in R \text {-alg }) .
$$

It is uniquely determined by the following universal property: for every $R$-functor $X$ there exists a bijection

$$
\xi=\xi_{X, Y^{\prime}}: \operatorname{Mor}_{S \text {-alg}}\left(X_{S}, Y^{\prime}\right) \stackrel{\sim}{\longrightarrow} \operatorname{Mor}_{R \text {-alg }}\left(X, \mathrm{R}_{S / R}\left(Y^{\prime}\right)\right)
$$

where $X_{S}$ is the $S$-functor obtained from $X$ by base change, thus satisfying $X_{S}(B)=X\left({ }_{R} B\right)$ for $B \in S$-alg. Here and sometimes in the following we write ${ }_{R} B$ to denote the $R$-algebra obtained from the $S$-algebra $B$ by restriction of scalars. The bijection $\xi$ is functorial in $X$ and $Y^{\prime}$. It maps $g \in \operatorname{Mor}_{S \text {-alg }}\left(X_{S}, Y^{\prime}\right)$ to the composition

$$
X(A) \stackrel{X\left(\mathrm{inc}_{1}\right)}{\longrightarrow} X\left({ }_{R}\left(A \otimes_{R} S\right)\right) \stackrel{g\left(A \otimes_{R} S\right)}{\longrightarrow} Y^{\prime}\left(A \otimes_{R} S\right)
$$

where $A \in R$-alg and inc $c_{1}$ is the $R$-algebra homomorphism

$$
\text { inc }_{1}=\text { inc }_{1, A}: A \longrightarrow R\left(A \otimes_{R} S\right), \quad a \mapsto a \otimes 1_{S} .
$$

We consider two special cases of (B.1.1). First, for $Y^{\prime}=X_{S}$ and $g=\operatorname{Id}_{X_{S}}$ we get the morphism

$$
\mathrm{j}=\mathrm{j}_{X}=\xi\left(\operatorname{Id}_{X_{S}}\right): X \longrightarrow \mathrm{R}_{S / R}\left(X_{S}\right)
$$

of $R$-functors, determined by $\mathrm{j}_{X}(A)=X\left(\mathrm{inc}_{1, A}\right), A \in R$-alg. Second, putting $X=\mathrm{R}_{S / R}\left(Y^{\prime}\right)$ in (B.1.1), there exists a unique morphism

$$
\mathrm{q}=\mathrm{q}_{Y^{\prime}}: \mathrm{R}_{S / R}\left(Y^{\prime}\right)_{S} \longrightarrow Y^{\prime}
$$

of $S$-functors satisfying $\xi\left(\mathrm{q}_{Y^{\prime}}\right)=\operatorname{Id}_{\mathrm{R}_{S / R}\left(Y^{\prime}\right)}$. For $B \in S$-alg we have $\mathrm{R}_{S / R}\left(Y^{\prime}\right)_{S}(B)=\mathrm{R}_{S / R}\left(Y^{\prime}\right)\left({ }_{R} B\right)=Y^{\prime}\left({ }_{R} B \otimes_{R} S\right)$ and so

$$
\mathrm{q}_{Y^{\prime}}(B): Y^{\prime}\left({ }_{R} B \otimes_{R} S\right) \longrightarrow Y^{\prime}(B) .
$$


In fact, $\mathrm{q}_{Y^{\prime}}(B)=Y^{\prime}\left(m_{B}\right)$ where $m_{B}$ is the $S$-algebra homomorphism

$$
m_{B}:\left({ }_{R} B\right) \otimes_{R} S \longrightarrow B, \quad b \otimes s \mapsto b s
$$

([CGP, A.5.7]). For $Y^{\prime}=X_{S}$ we now have constructed morphisms

$$
X_{S} \stackrel{\mathrm{j}_{X, S}}{\longrightarrow} \mathrm{R}_{S / R}\left(X_{S}\right)_{S} \stackrel{\mathrm{q}_{X_{S}}}{\longrightarrow} X_{S}
$$

between $S$-functors. Untangling the constructions above, we find

$$
\mathrm{q}_{X_{S}} \circ \mathrm{j}_{X, S}=\operatorname{Id}_{X_{S}}
$$

because $B \stackrel{\text { inc }_{1, B}}{\longrightarrow} R B \otimes_{R} S \stackrel{m_{B}}{\longrightarrow} B$ equals $\operatorname{Id}_{B}$.

B.2. Cohomology and restriction. Let $G$ be a flat $R$-group sheaf. We denote by

$$
H^{1}(R, G)=H_{\text {fppf }}^{1}(R, G)
$$

the pointed set of isomorphism classes of $G$-torsors over $\operatorname{Spec}(R)$ in the flat topology. Let $S \in R$-alg. The base change $X_{S}=X \times_{\operatorname{Spec}(R)} \operatorname{Spec}(S)$ of a $G$-torsor $X$ is a $G_{S}$-torsor, giving rise to the restriction map

$$
\text { res }=\operatorname{res}_{S / R, G}: H^{1}(R, G) \rightarrow H^{1}\left(S, G_{S}\right), \quad[X] \mapsto\left[X_{S}\right] .
$$

A homomorphism $f: G \rightarrow H$ of flat $R$-group sheaves induces a map in cohomology

$$
f_{*}: H^{1}(R, G) \rightarrow H^{1}(R, H), \quad[X] \mapsto\left[X \wedge^{G} H\right]
$$

where $X \wedge^{G} H=\left(X \times_{\operatorname{Spec}(R)} H\right) / G$ is the contracted product with respect to the $G$-action on $H$ via $f$. Contracted products are special cases of fppf quotients and as such allow base change [GM, (4.30)]. Hence $\left(X \wedge^{G} H\right)_{S}$ and $X_{S} \wedge^{G_{S}} H_{S}$ are isomorphic $H_{S}$-torsors, giving rise to a commutative diagram

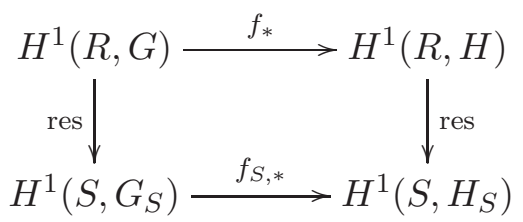

of pointed sets. It is known that $H=\mathrm{R}_{S / R}\left(G_{S}\right)$ is again a flat $R$-group sheaf if $G$ is so and that the maps

$$
\mathrm{j}: G \rightarrow \mathrm{R}_{S / R}\left(G_{S}\right) \text { and } \mathrm{q}: \mathrm{R}_{S / R}\left(G_{S}\right)_{S} \rightarrow G_{S}
$$

of B.1 are homomorphisms of $R$-group sheaves. Hence, passing to cohomology, the maps in the diagram are well-defined:

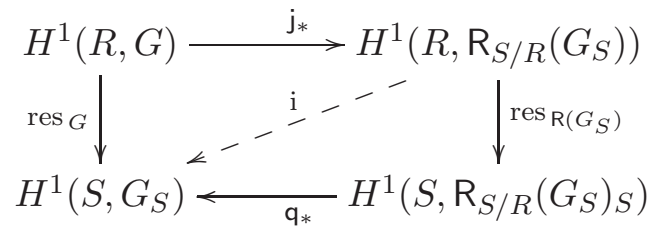


We claim that (B.2.2) is a commutative diagram. Indeed, since q०j. $\mathrm{j}_{S}=\operatorname{Id}_{G_{S}}$ by (B.1.2), this follows from commutativity of (B.2.1):

$$
\operatorname{res}_{G}=\mathbf{q}_{*} \circ\left(\mathrm{j}_{S}\right)_{*} \circ \operatorname{res}_{S / R, G}=\mathrm{q}_{*} \circ \operatorname{res}_{\mathrm{R}_{S / R}\left(G_{S}\right)} \circ \mathbf{j}_{*}=\mathrm{i} \circ \mathrm{j}_{*} .
$$

The map $\mathrm{i}=\mathrm{q}_{*} \circ \operatorname{res}_{\mathrm{R}_{S / R}\left(G_{S}\right)}$ is injective by [SGA3, XXIV.8.2].

In particular, assume that $G$ is an abelian affine $R$-group scheme. Then so are $G_{S}, \mathrm{R}_{S / R}\left(G_{S}\right)$ and $\mathrm{R}_{S / R}\left(G_{S}\right)_{S}$. Moreover, the cohomology sets and maps used in (B.2.2) are abelian groups and group homomorphisms respectively. Since $\mathrm{i}$ is injective, we get from (B.2.3) that

$$
\operatorname{Ker}\left(\operatorname{res}_{G}\right)=\operatorname{Ker}\left(\mathbf{i} \circ \mathbf{j}_{*}\right)=\operatorname{Ker}\left(\mathbf{j}_{*}\right) .
$$

B.3. Deligne trace homomorphism. Let $S \in R$-alg be locally free of finite rank $d \in \mathbb{N}_{+}$, i.e., the $R$-module $S$ is projective of constant rank $d$, and let $G$ be an abelian affine $R$-group scheme. We then have Deligne's trace homomorphism

$$
\operatorname{tr}: \mathrm{R}_{S / R}(G) \rightarrow G, \quad \text { satisfying } \operatorname{tr} \circ \mathrm{j}=\times d,
$$

where $\times d: G \rightarrow G$ is the group homomorphism given on $T$-points, $T \in$ $R$-alg, by $g \mapsto g^{d}\left[\mathrm{SGA}_{3}\right.$, XVII, 6.3.13-6.3.15]. It induces an endomorphism

$$
(\times d)_{*}: H^{1}(R, G) \longrightarrow H^{1}(R, G)
$$

given by the analogous formula. Since $(\times d)_{*}=\operatorname{tr}_{*} \circ \mathbf{j}_{*}$ by (B.3.1), we obtain from (B.2.4) that

$$
\operatorname{Ker}\left(\operatorname{res}_{S / R, G}\right)=\operatorname{Ker}\left(\mathrm{j}_{*}\right) \subset \operatorname{Ker}\left((\times d)_{*}\right) .
$$

In particular, this implies the following.

B.4. Lemma. In the setting of B.3 assume that $\times d: G \rightarrow G$ is an isomorphism. Then the restriction homomorphism $\operatorname{res}_{S / R, G}: H_{\mathrm{fpp}}^{1}(R, G) \rightarrow$ $H_{\text {fppf }}^{1}(S, G)$ is injective.

Example. Let $G$ be the constant $R$-group scheme $\mathbb{Z} / 2 \mathbb{Z}$, which is the automorphism group scheme of a quadratic étale $R$-algebra $A$, and let $d$ be odd. Then Lemma B.4 applies and in particular shows that $A$ is split, i.e., $A=R \times R$, if and only if $A_{S}$ is split.

Acknowledgments. We thank M. Ojanguren and the referee for helpful comments on an earlier version of this paper.

\section{REFERENCES}

[AG] S. Alsaody, P. Gille, Isotopes of octonion algebras, $\mathrm{G}_{2}$-Torsors and triality, Adv. Math. 343 (2019), 864-909.

[Ba] R. Baeza, Quadratic Forms over Semilocal Rings, Lecture Notes in Mathematics 655 (1978).

[BFP] E. Bayer-Fluckiger, U. A. First, and R. Parimala, On the Grothendieck-Serre Conjecture for Classical Groups, arXiv:1911.07666

[BLR] S. Bosch, W. Lütkebohmert, M. Raynaud, Néron models, Ergebnisse der Mathematik und ihrer Grenzgebiete, Band 21, Springer-Verlag 1990. 
[Co1] B. Conrad, Reductive group schemes, in Autour des schémas en groupes, vol. I, Panoramas et Synthèses 42-43, Soc. Math. France 2014.

[Co2] Standard parabolics subgroups. Theory and Examples, http://math.stanford.edu/ conrad/249BW16Page/handouts/stdpar.pdf

[CGP] B. Conrad, O. Gabber, and G. Prasad, Pseudo-reductive groups, second edition. New Mathematical Monographs 26, Cambridge University Press, Cambridge 2015.

[DG] M. Demazure and P. Gabriel, Groupes algébriques, North-Holland (1970).

[EGA] A. Grothendieck (avec la collaboration de J. Dieudonné), Eléments de Géométrie Algébrique, Publications mathématiques de l'I.H.É.S. no. 4, 8, 11, 17, 20, 24, 28, 32, 1960-1967.

[EKM] R. Elman, N. Karpenko, and A. Merkurjev, The algebraic and geometric theory of quadratic forms, Amer. Math. Soc. Colloq. Publ. 56, Amer. Math. Soc., Providence, RI, 2008.

[Fo] T. J. Ford, Separable Algebras, Graduate Studies in Mathematics 183, Amer. Math. Soc, Provident, RI, (2017).

[GM] G. van der Geer and B. Moonen, preliminary version of a book on Abelian Varieties, https://www.math.ru.nl/ bmoonen/research.html\#lecturenotes

[Ho1] D. W. Hoffmann, Diagonal forms of degree $p$ in characteristic $p$, Algebraic and arithmetic theory of quadratic forms, 135--183, Contemp. Math., 344, Amer. Math. Soc., Providence, RI, 2004.

[Ho2] Similarity of quadratic and symmetric bilinear forms in characteristic 2, Indagationes Mathematicae (2020), https://doi.org/10.1016/j.indag.2020.08.008.

[Kne] M. Knebusch, Isometrien über semilokalen Ringen, Math. Z. 108 (1969), 255268.

[Knu] M.-A. Knus, Quadratic and Hermitian Forms over Rings, Grundlehren der mathematischen Wissenschaften 294 (1991), Springer.

[Lag] A. Laghribi, The norm theorem for totally singular quadratic forms, Rocky Mountain J. Math. 36 (2006), 575-592.

[Lam] T. Y. Lam, An Introduction to Quadratic Forms over Fields, Graduate Studies in Mathematics 67, Amer. Math. Soc., Providence, RI, 2005.

[Lo] O. Loos, Generically algebraic Jordan algebras over commutative rings, J. Algebra 297 (2006), 474-529.

[PR] I. Panin and U. Rehman, A variant of a theorem by Springer, St. Petersburg Math. J. 19 (2008), 953-959.

[PP] I. Panin and K. Pimenov, Rationally isotropic quadratic spaces are locally isotropic: II, Doc. Math. 2010, Extra vol.: Andrei A. Suslin sixtieth birthday, $515--523$.

[Pe] H. P. Petersson, A survey on Albert algebras, Transform. Groups 24 (2019), 219--278.

[Sa] C. H. Sah, Symmetric bilinear forms and quadratic forms, J. Algebra 20 (1972), 144-160.

[Sch] W. Scharlau, Quadratic and Hermitian Forms, Grundlehren der Mathematischen Wissenschaften 270, Springer-Verlag Berlin 1985.

[Scu] S. Scully, The Artin-Springer Theorem for quadratic forms over semi-local rings with finite residue fields, Proc. Amer. Math. Soc. 146 (2018), 1-13.

[SGA3] Séminaire de Géométrie algébrique de l'I.H.E.S., 1963-1964, schémas en groupes, dirigé par M. Demazure et A. Grothendieck, Lecture Notes in Math. 151-153. Springer (1970).

[SGA4 $4_{3}$ Théorie des topos et cohomologie étale des schémas, Tome 3 (French), Séminaire de Géométrie Algébrique du Bois-Marie 1963-1964 (SGA 4). Dirigé par M. Artin, 
A. Grothendieck et J. L. Verdier. Avec la collaboration de P. Deligne et B. SaintDonat. Lecture Notes in Mathematics 305, Springer-Verlag, Berlin-New York, 1973.

[Sp] T. A. Springer, Sur les formes quadratiques d'indice zéro, C. R. Acad. Sci. Paris 234 (1952), 1517-1519.

[St] The Stacks Project Authors, stacks project, http://stacks.math.columbia.edu/

UMR 5208 du CNRS - Institut Camille Jordan - Université Claude Bernard Lyon 1, 43 Boulevard du 11 nOVEMBRE 1918, 69622 Villeurbanne CEdeX - France Email address: gille@math.univ-lyon1.fr

Department of Mathematics and Statistics, University of Ottawa, Ottawa, Ontario, Canada, K1N 6N5

Email address: neher@uottawa.ca 